\title{
BMJ Open Quantifying the prevalence of frailty in English hospitals
}

\author{
J Soong,,$^{1,2}$ AJ Poots, ${ }^{1}$ S Scott, ${ }^{3}$ K Donald, ${ }^{3}$ T Woodcock, ${ }^{1}$ D Lovett, ${ }^{1}$ D Bell ${ }^{1}$
}

To cite: Soong J, Poots AJ, Scott S, et al. Quantifying the prevalence of frailty in English hospitals. BMJ Open 2015;5:e008456. doi:10.1136/bmjopen-2015008456

- Prepublication history and additional material is available. To view please visit the journal (http://dx.doi.org/ 10.1136/bmjopen-2015008456).

Received 15 April 2015 Revised 31 July 2015 Accepted 21 August 2015

\section{SLinked}

- http://dx.doi.org/10.1136/ bmjopen-2015-008457

\section{CrossMark}

\section{${ }^{1}$ NIHR CLAHRC Northwest London, Imperial College London, London, UK \\ ${ }^{2}$ Royal College of Physicians, London, UK \\ ${ }^{3}$ Oliver Wyman, London, UK}

Correspondence to

Dr J Soong;

johnsoong@imperial.ac.uk

\section{ABSTRACT}

Objectives: Population ageing has been associated with an increase in comorbid chronic disease, functional dependence, disability and associated higher health care costs. Frailty Syndromes have been proposed as a way to define this group within older persons. We explore whether frailty syndromes are a reliable methodology to quantify clinically significant frailty within hospital settings, and measure trends and geospatial variation using English secondary care data set Hospital Episode Statistics (HES).

Setting: National English Secondary Care Administrative Data HES.

Participants: All 50540141 patient spells for patients over 65 years admitted to acute provider hospitals in England (January 2005-March 2013) within HES.

Primary and secondary outcome measures: We explore the prevalence of Frailty Syndromes as coded by International Statistical Classification of Diseases, Injuries and Causes of Death (ICD-10) over time, and their geographic distribution across England. We examine national trends for admission spells, inpatient mortality and 30-day readmission.

Results: A rising trend of admission spells was noted from January 2005 to March 2013(daily average admissions for month rising from over 2000 to over 4000). The overall prevalence of coded frailty is increasing (64 559 spells in January 2005 to 150085 spells by Jan 2013). The majority of patients had a single frailty syndrome coded $(10.2 \%$ vs total burden of $13.9 \%$ ). Cognitive impairment and falls (including significant fracture) are the most common frailty syndromes coded within HES. Geographic variation in frailty burden was in keeping with known distribution of prevalence of the English elderly population and location of National Health Service (NHS) acute provider sites. Overtime, in-hospital mortality has decreased (>65 years) whereas readmission rates have increased (esp.>85 years).

Conclusions: This study provides a novel methodology to reliably quantify clinically significant frailty. Applications include evaluation of health service improvement over time, risk stratification and optimisation of services.

\section{INTRODUCTION}

People are living longer. At present, it is estimated that $16.1 \%$ of the European population is over the age of 65 years ( $>65$ years),

\section{Strengths and limitations of this study}

- This study is the first to attempt to use frailty syndromes as an operational definition within an English secondary care data set.

- The methodology uses whole population routinely collected data, with robust trend analysis examining coding reliability.

- This study is a retrospective analysis reliant on the accuracy, reliability and retrospective nature of coding within Hospital Episode Statistics.

and this number is expected to rise to $22 \%$ by 2031. In the developed world, the increase is greatest in those over 80 years, and this equates to approximately 3 million people in the UK. ${ }^{2}$ In health terms patients $>65$ years now constitute two-thirds of the general hospital population, account for $40 \%$ of all hospital bed days and $65 \%$ of National Health Service (NHS) spend. ${ }^{3}$ Recent analysis suggests population ageing contributes directly to the increase in emergency admissions to hospitals. ${ }^{4}$

Associated with this demographic shift there has been an increase in comorbid chronic disease, functional dependence, disability, poorer quality of life and higher health care costs. ${ }^{56}$ Patients in this category are often considered frail. Currently, there is no universally agreed operational definition for frailty. ${ }^{7}$ Frailty has been described as a clinical phenotype or a biophysical syndrome of accumulated deficit (frailty index). Phenotypic models describe frailty as specific clinical syndrome encompassing a cluster of characteristics, namely unintentional weight loss, exhaustion, weakness, slowness and low physical activity. ${ }^{8}$ The frailty index is characterised by decreased resistance to stressors resulting from the accumulation of deficit across multiple physiological systems, culminating in an increased risk of adverse outcomes. ${ }^{910}$ Methodologies to reliably identify the 'frail' at-risk cohort within secondary care, both at patient and population level, are a current research priority. ${ }^{11-13}$ 
In clinical practice the terms Geriatric Giants, ${ }^{14}$ Geriatric Syndromes ${ }^{15} 16$ or Frailty Syndromes ${ }^{17}$ are often used to describe clinically vulnerable group within the elderly. They likely represent high order clinical manifestations of multifactorial processes resultant from the accumulation and interaction of deficits and environmental factors. They include cognitive impairment, falls, mobility problems, pressure ulcers and incontinence. These syndromes, more prevalent in the elderly, confer a higher risk of death, ${ }^{8}$ institutionalisation, ${ }^{18}$ disability and poor quality of life. ${ }^{15}$ They are arguably the consequences of frailty, or the manifestation of clinically significant frailty. ${ }^{19}$ Current National guidelines for the care of the older person in acute care recommend using frailty syndromes as a possible methodology to assess for frailty. ${ }^{17} 20$

In this study, we measure the trends for all hospital admissions, in-hospital death and readmissions for those over 65 years. We describe Frailty Syndromes ${ }^{17}{ }^{20}$ as an operational definition within the English secondary care data set Hospital Episode Statistics (HES) in order to examine the frailty burden between 2005 and 2012. In addition we describe the geospatial variation of frailty in English secondary healthcare settings. We compare our results with the existing literature on frailty prevalence and discuss possible applications of this methodology.

\section{METHODS}

\section{Data sources}

HES is a national administrative database containing patient-level records of all admissions to NHS hospitals in England. ${ }^{21}$ It has high levels of data completeness and rigorous data cleaning processes to ensure data quality. Each record in HES corresponds to a finished consultant episode, during which a patient is under the care of an individual consultant. These episodes were aggregated into hospital spells covering a patient's total length of stay in a hospital (ie, a hospital admission) using established methodology. ${ }^{22}$

HES contains 20 fields per record for diagnoses codes that are defined using the tenth revision of the International Statistical Classification of Diseases, Injuries and Causes of Death (ICD-10). The first of these is the primary diagnosis, with the rest available for coding of comorbidities or complications. HES does not contain present-on-admission flags. We reviewed HES for ICD-10 diagnostic codes that could be grouped for frailty syndromes (see online supplementary appendix 1) in all 20 fields. We included only inpatients at acute non specialist hospital trusts, with elective and non-elective admissions for those 65 years and over $>65$ years. We excluded hyper-specialist hospitals and mental health units as they have a very different case-mix and data quality. ${ }^{23}$ Thus, we defined frailty as the presence of at least one frailty syndrome and within the cohort of patients greater than 65 years old.

Annual trend profiles were created for the grouped ICD-10 diagnostic codes from January 2005 to March
2013 to determine coding reliability and shifts (see online supplementary appendix 2). The spells were aggregated both by age-band (65-74; 75-84; >85 years) and monthly. Monthly data are visualised as simple line plots in the first instance. Office of National Statistics (ONS) databases were queried for population size estimates or census data where available.

\section{Study population}

All hospital admissions for $>65$ years to English acute trusts between January 2005 and March 2013 ( $\mathrm{N}=50540141$ patient spells) were available for analysis.

\section{Temporal analysis}

To analyse the variation present in these time-series data, statistical process control is used to separate special cause variation (signal) from common cause variation, an inherent property of all systems. The XmR chart is used as it is a method that is not dependent on data distributions or underlying assumptions. ${ }^{24}$ When analysing count data, daily averages for months were calculated to correct for unequal 'areas of opportunity'; for example, a count of February admissions will be lower by virtue of fewer days in February, and daily averages account for the difference in available days. For percentage data, such a correction is attained through division by the denominator-all spells and all spells with frailty. Adjustments for seasonal variation are made, and seasonalised reference lines are plotted, for more natural interpretation of the charts. In this work, a standard rule set for detection of signal is adopted, using Microsoft Excel to construct the charts. ${ }^{24}$

\section{Geospatial analysis}

Geo-location is the identification of real-world geographic location of an object. Postcodes of provider sites were used to geo-locate sites, and map elements were derived from open source data provided by Office for National Statistics. Geo-locations aggregated to Primary Care Trust (PCT) level were attached to counts of frailty syndromes for patients $>65$ years admitted to NHS acute providers in 2012 as this is the applicable unit for these data. Choropleths are thematic maps that shade or colour areas to represent classified values of specific phenomena. ESRI ArcMap V.10.2 software was used to create a choropleth map. Annual trend profiles for inpatient mortality and non-elective readmission within 30 days were plotted. This temporal range of April 2006 to December 2012 was selected due to changes in structure of health geographies within England in 2006, ${ }^{26}$ and to allow a sufficient follow up period to more accurately reflect the clinical outcomes listed above.

\section{RESULTS}

Between January 2005 and March 2013, there was a rising trend with daily average admissions for month increasing from over 2000 to over 4000 (figure 1A). 

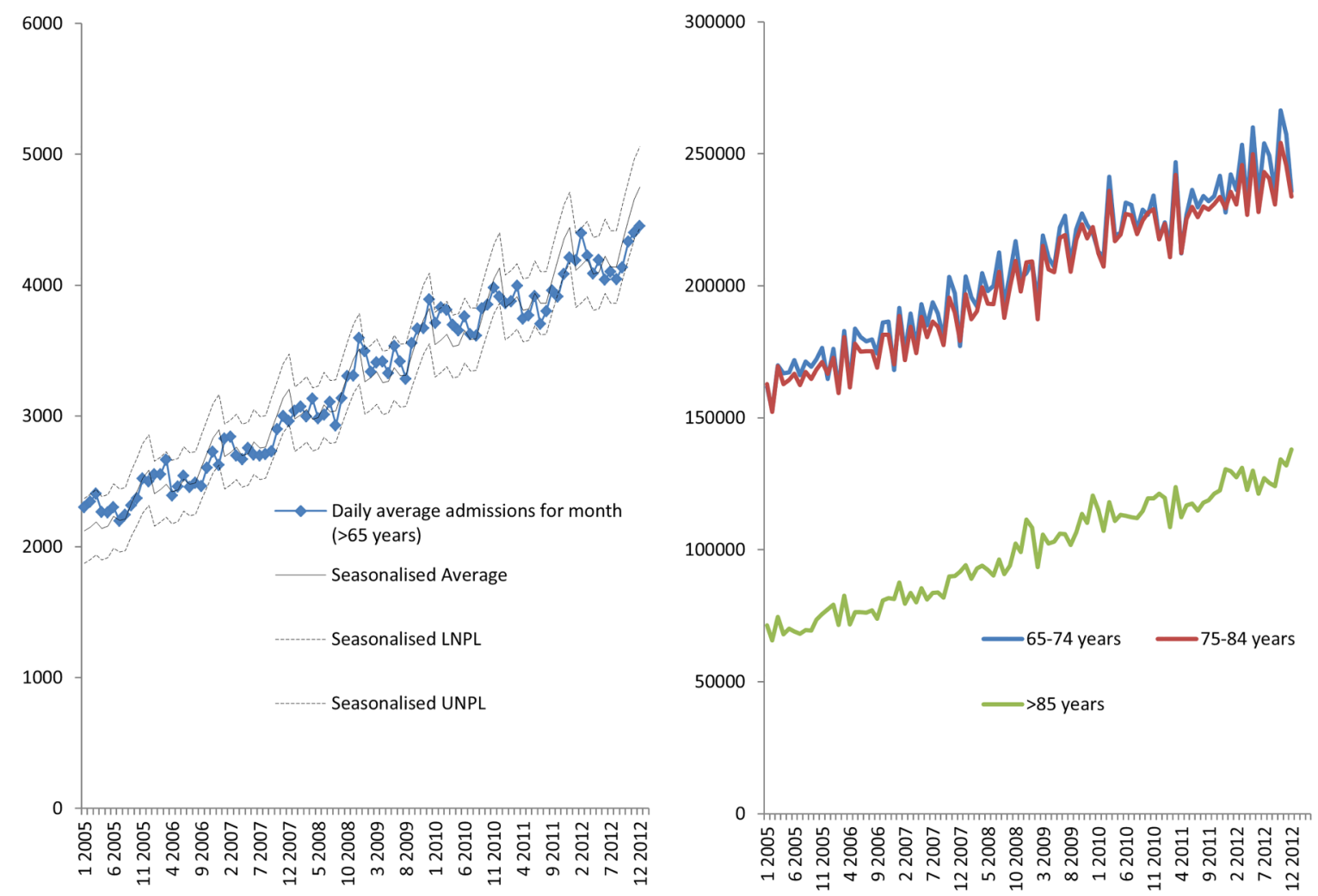

Figure 1 (A) Daily average admission spells for month and percentage total frailty burden for England NHS acute trusts. (B) The number and percentage of spells for patients $>65$ years by age-band admitted to English acute providers. LNPL, Lower Natural Process Limits; NHS, National Health Service; UNPL, Upper Natural Process Limits.

There has been an increase in all age bands over this period, 65-74 increasing from 161641 to 235 756, 75-84 increasing from 162817 to 233870 and $>85$ increasing from 71396 to 137991 (figure 1B). The relative proportion of total admissions has remained constant each age band at $40 \%, 40 \%$ and $20 \%$, respectively. Examination of ONS data, (see online supplementary appendix 4) finds that in the general UK population the number of $>64$ years old in the population increased from 8031000 in 2005 , to 905179 in 2013. In 2005, the 65$74 \mathrm{~s}$ represented $52 \%$ of those $>65$-year; in 2013 it was $54 \%$; $75-84$ s were $36 \%$ and $33 \%$ 2005-2013; and $>85 \mathrm{~s}$ were $12 \%$ and $13 \%$.

Analysis of trends shows that the coded overall frailty burden, based on the coding of at least one frailty syndrome, has increased from $12 \%$ to $14 \%$ between January 2005 and March 2013. There is evidence of seasonal peaks during winter, partly explained by similar patterns in admission spells (figure 2).

The coding of the frailty syndromes has increased between 2005 and 2013. Most patients had one frailty syndrome coded (figure 3) and the most common frailty syndromes described between 2005 and 2013 were cognitive impairment and falls (including significant fracture) with cognitive impairment increasing to the same levels as falls representing approximately $10 \%$ of all spells in the those $>65$ years. Anxiety and/or depression has increased particularly from 2010 (2.4\%) to
$2013(>4 \%$ ) (figure 4). There is a persistent and steady rise in coding for mobility problems.

Evaluating the frailty syndromes individually, the very elderly (>85 years) represent between $40 \%$ and $50 \%$ of the spells coded for that syndrome, with rising trend. The exception to this was anxiety and/or depression syndrome, which exhibited a rising trend in the 65-74 s, and the 75-84 $\mathrm{s}$ accounted for the largest group (see online supplementary appendix 3). Age-band stratification shows that cognitive impairment and falls in agebands $>85$ years and 75-84 years account for a large majority of coded frailty syndromes within this cohort. These four groups accounted for $60.2 \%$ of frailty syndromes coded over this time period $(\mathrm{N}=7399671)$

Geographic variation in the frailty burden across admission spells in England was seen based on the 2012 HES data (figure 5). For patients $>65$-year admitted to England Acute providers, the highest levels of frailty are seen in the Northeast, Central and South Coast. The top five PCTs for highest admissions numbers are Nottingham City, Halton \& St Helens, Warrington, Waltham Forrest and Wolverhampton city.

Between April 2006 to December 2012, 1160299 $(3.4 \%)$ spells were associated with inpatient mortality, though a decreasing trend is observed for example, April 2006 ( $\mathrm{N}=15042)$ to April 2012( $\mathrm{N}=14437)$ (figure $6 \mathrm{~A})$. Non-elective re-admission rates within 30 days of discharge have increased for all admissions $>65$ years 


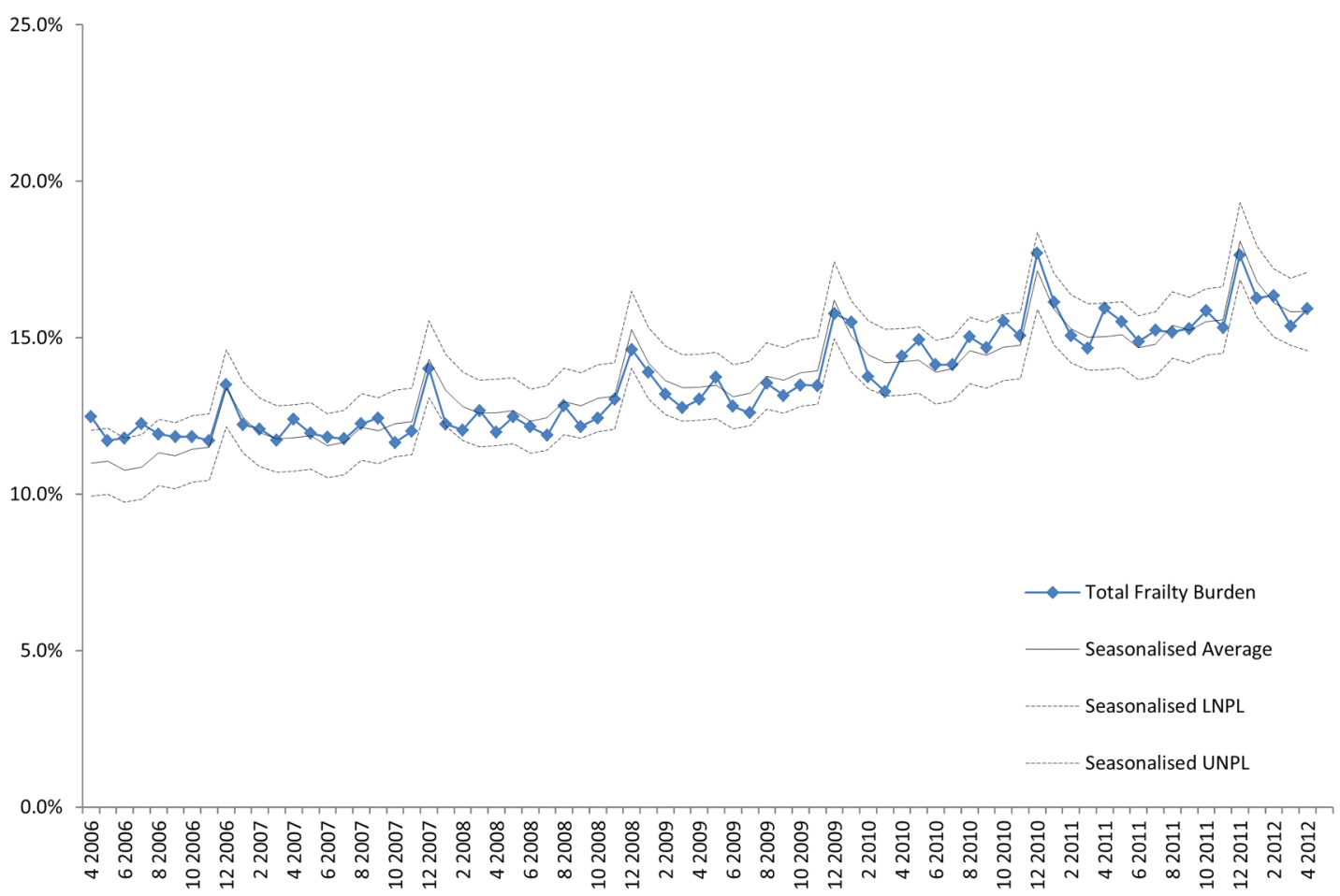

Figure 2 The percentage of admissions to English acute providers coded with at least one frailty syndrome. LNPL, Lower Natural Process Limits; UNPL, Upper Natural Process Limits.

from approximately $11-12 \%$ (figure $6 \mathrm{~B}$ ). The rates of readmission increased across the age bands $>65$ years $(10 \%), 75-84(12 \%)$ and $>85 \quad(14 \%)$. Though the overall number of very elderly ( $>85$ years $)$ with non-elective 30-day readmission is lower than the other two age-bands, they have more readmissions (figure 7).

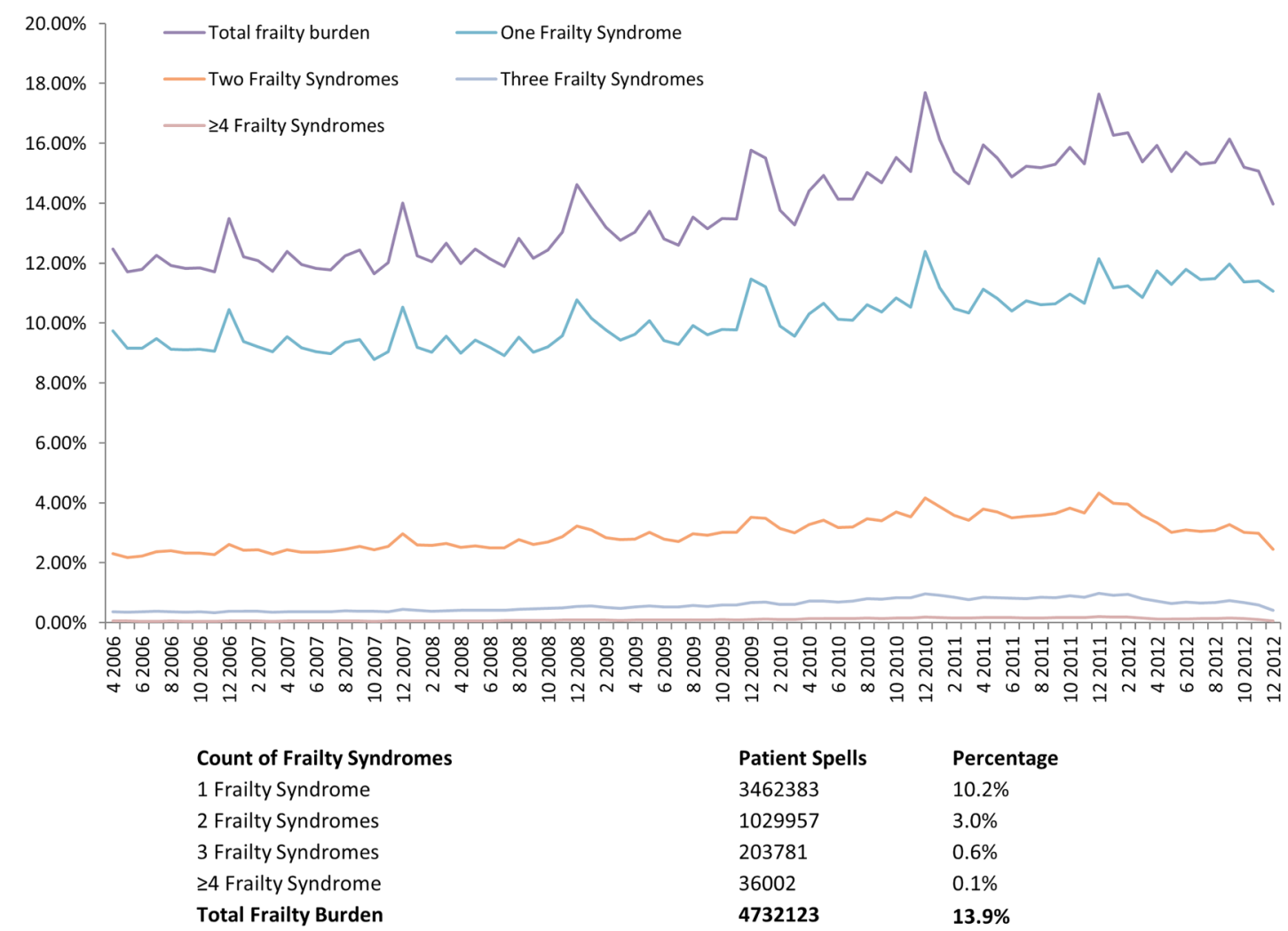

Figure 3 Trends for the prevalence of count of frailty syndromes and total frailty burden for patients $>65$ years admitted to NHS acute provider hospitals between April 2006 and December 2012. NHS, National Health Service. 


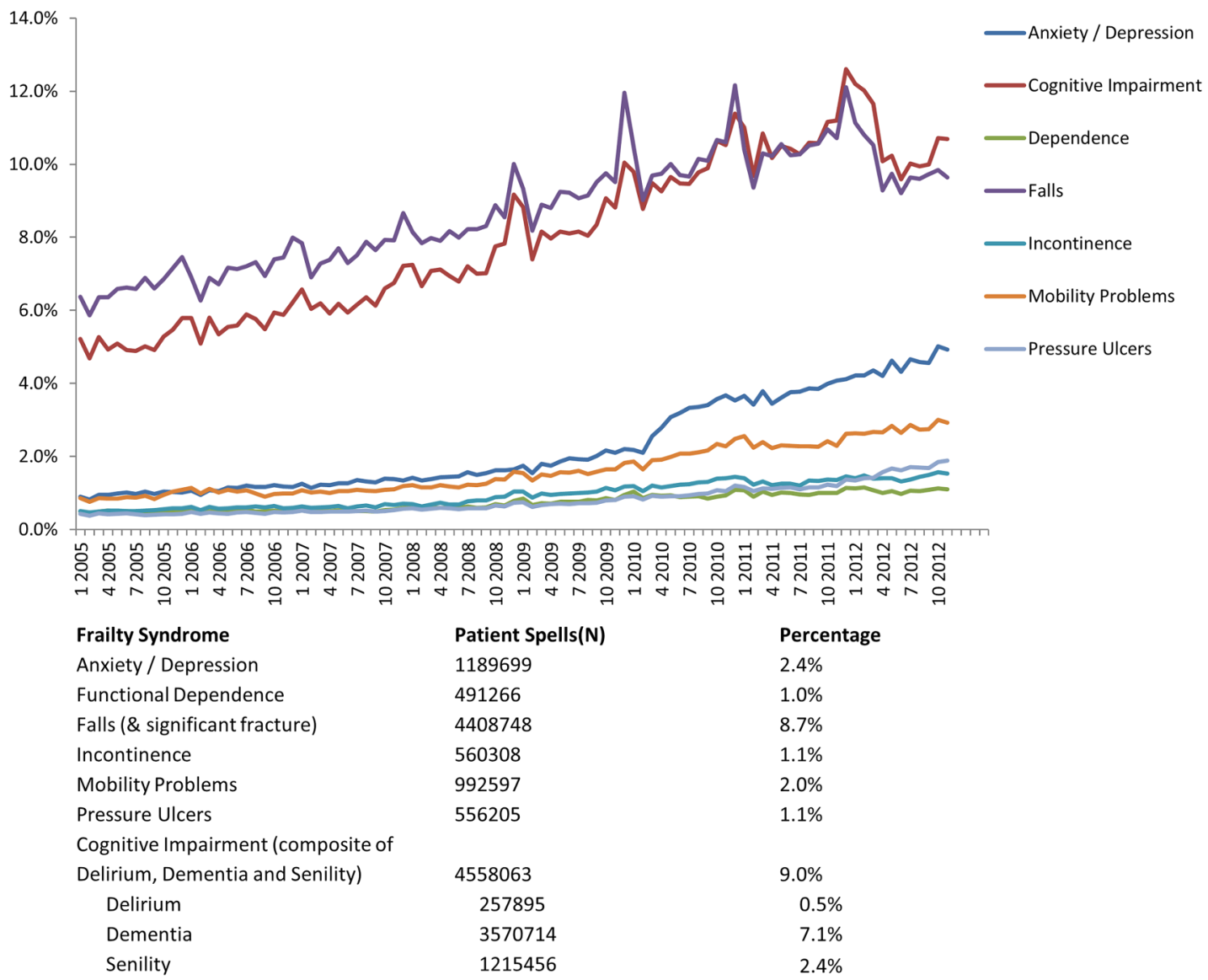

Figure 4 Trends for the prevalence of frailty syndromes for patients $>65$ years admitted to NHS acute provider hospitals between January 2005 and March 2013. NHS, National Health Service.

\section{DISCUSSION}

Frailty is often defined as a clinical state in which there is an increase in an individual's vulnerability for adverse events and harm when exposed to a stressor. ${ }^{25}$ It is distinct but related to disability and comorbidity. ${ }^{26}{ }^{27}$ Some approaches to the measurement of frailty have been characteristically biophysical with emphasis on detection of the consequences of sarcopaenia and chronic inflammation-malnutrition. ${ }^{8}$ Another approach is to measure frailty in relation to the clinical consequences of accumulated loss and insufficiency in ageing individuals(ie, the relationship to mortality and adverse outcomes). ${ }^{28}$ Both approaches appear complementary ${ }^{29}$ and overlap, though not completely. ${ }^{30}$ Frailty measurement is problematic in the acute care setting. High levels of disease acuity on top of chronic multimorbidity, multidimensional complexity and diagnostic uncertainty are challenging for healthcare systems, with increasing evidence and concern for compromised patient safety, quality of care and experience. ${ }^{31-34}$

We have examined the prevalence of frailty syndromes within English HES data from both a temporal and geospatial point of view. Temporal analysis, it allows us to observe shifts in diagnostic coding, and observe trend in signal changes over time. Spatial analysis allows us to explore geographic heterogeneity of frailty syndrome prevalence, with consequent implications for service provision and equity of care.

Comparison with ONS data, the corresponding admissions to English acute providers for patients with frailty syndromes is larger than might be expected by demographic shift associated with ageing. Additionally, 75$84 \mathrm{~s}$ make up approximately one-third of the population of those over 65 years, but have $40 \%$ of the admissions, and $>85 \mathrm{~s}$ are approximately $13 \%$ of the population of those over 65 years but have $20 \%$ of the admissions.

This study has focused on patients admitted to hospital $>65$ years in England to better understand the impact of frailty syndromes. To the authors knowledge, this is the first study to examine the prevalence of frailty syndromes for patients $>65$ years across England. This study confirms increasing number of $>65$ years admitted to hospital (elective and non-elective). The relative burden of coded frailty syndromes has increased over this period with cognitive impairment increasing to similar levels to falls. Anxiety and/or depression is also increasing in this group.

When complex systems fail (biological or otherwise), high-order functions can be first disrupted. ${ }^{35}$ Frailty syndromes represent the clinical manifestation of highorder disruption, providing a useful clinical marker of multidimensional deficit accumulation. The overall 


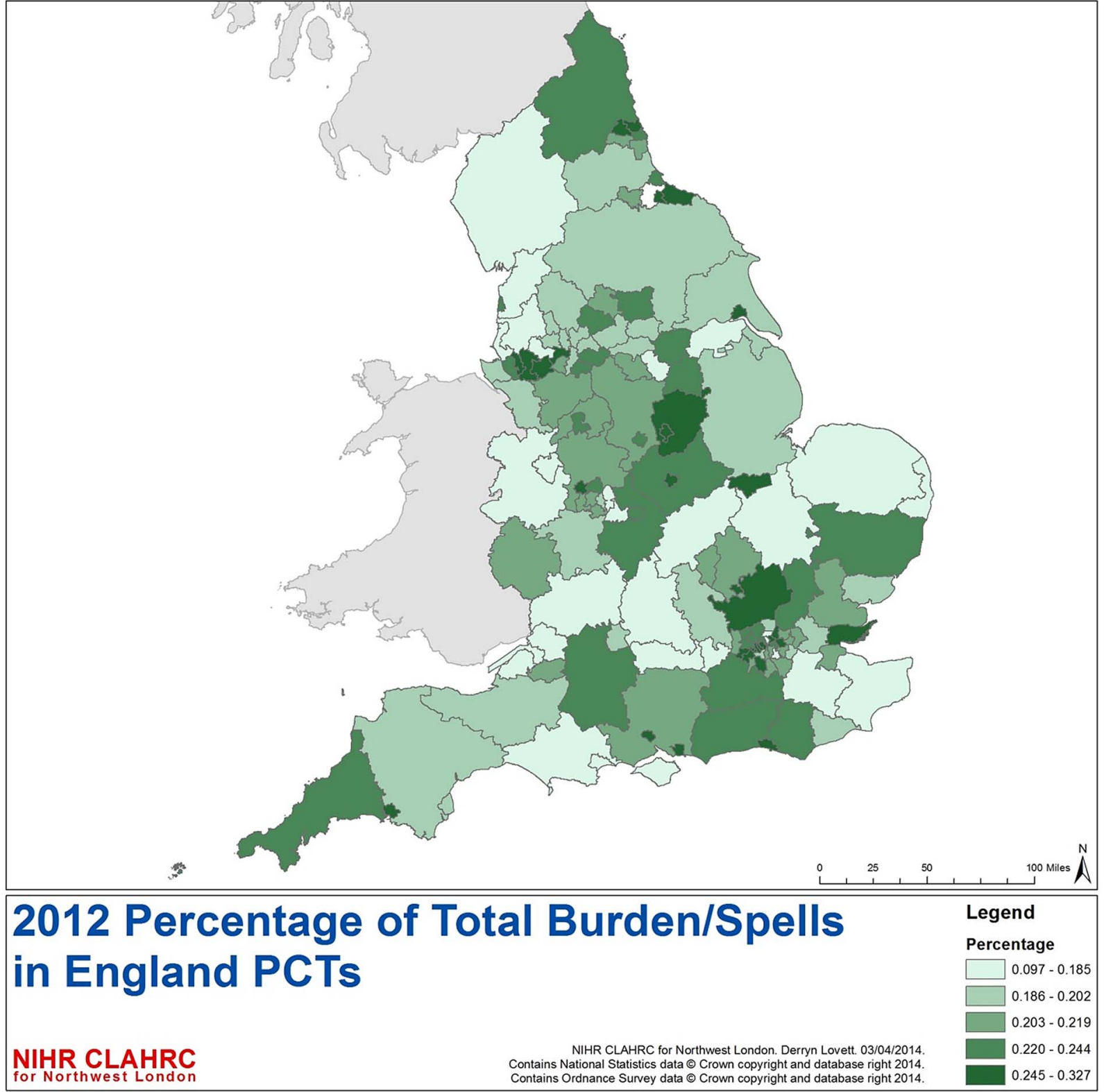

Figure 5 Percentage of spells for patients >65 years with admission to NHS acute trusts with at least one frailty syndrome by PCT by quintiles (numerator=admission spells with at least one frailty syndrome; denominator=total admission spells to NHS acute trusts within English PCT). NHS, National Health Service; PCT, Primary Care Trust.

prevalence rate of frailty syndromes found in this study is $13.9 \%$. Between 2005 and 2013, though there has been an increase in the numbers of patients admitted $>65$ years, the percentage by age band has remained stable, thus not suggesting major drift towards older age groups within the older population. However, within the $>65$ years group, frailty syndromes are more prevalent with the older age bands.

Prevalence rates of frailty vary depending on population and operational definition used in reported studies. Reported prevalence in community dwelling adults varies tremendously (from $4.0 \%$ to $59.1 \%$ ). ${ }^{36} \mathrm{~A}$ recent systematic review reported pooled frailty prevalence across 21 community dwelling study cohorts as $10.7 \%$
$(\mathrm{N}=61500) .{ }^{36}$ The recent Survey of Health, Ageing and Retirement in Europe (SHARE) study reported frailty prevalence as $4.1 \%$ in community dwelling adults $>50$ years $(\mathrm{N}=16584)$ in 10 European countries (prevalence of $17 \%$ in those over 65 years). ${ }^{37}$ In the UK, the Hertfordshire Cohort Study ${ }^{38}$ reported an overall prevalence of $6.3 \%$ for 638 community dwelling 64-74-year-olds, while the English Longitudinal study of ageing ${ }^{39}$ reported a prevalence of $8 \%$ and $13 \%$ for 3055 community dwelling over 65-year-olds (using the Phenotype ${ }^{8}$ and Frailty Index ${ }^{10}$ definitions, respectively).

The prevalence of inpatient frailty in our study was lower than expected from smaller reported clinical studies within secondary care (range $24.7 \%-80 \%$ ): 

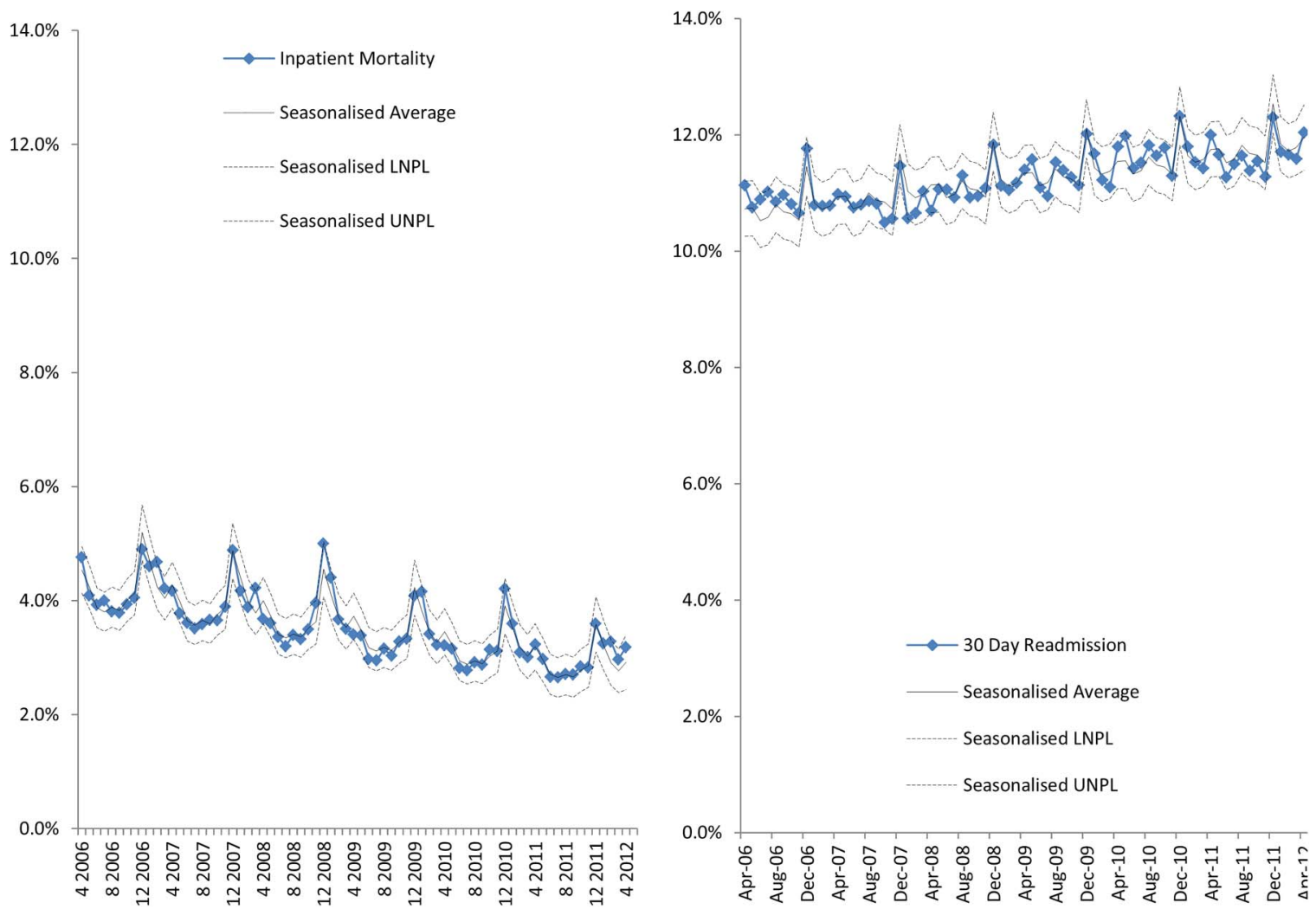

Figure 6 (A) Percentage of spells with inpatient mortality admitted to English providers and (B) non-elective 30-day readmission in patients $>65$ years admitted to English acute providers.

$(\mathrm{n}=220>70$ years admitted to acute geriatric ward from Emergency department, ${ }^{40}(\mathrm{n}=6701) 40 \%$ (Phenotype) and $32.5 \%\left(\mathrm{SOF}_{;}{ }^{11} \quad(\mathrm{n}=1388>70\right.$ years admitted to cardiology service, ${ }^{42}(\mathrm{n}=900827 \%$ (Phenotype) and 63\% (Frailty Scale $\left.^{43}\right) ;(\mathrm{n}=298>75$ years admitted to five different specialist wards, 50-80\% (Groningen Frailty Index $\left.{ }^{44}\right)$; $(n=307$ $>75$ years with diagnosed non-ST elevation myocardial infarction, ${ }^{46} 48.5 \%\left(\mathrm{n}=2305>65\right.$ years Clinical Frailty Scale $\left.{ }^{47}\right)$; $\left(\mathrm{n}=752\right.$ medical inpatients $>75$ years. ${ }^{48}$ In the UK, two recent studies ${ }^{12} 13$ reported frailty prevalence for $\mathrm{n}=667$ patients > 70 years admitted to Acute Medical Units(AMU) at $69 \%$ (ISAR, ${ }^{49} 17.9 \%$ (Phenotype), $66.4 \%$ (SOF), $24.9 \%$ (Avila-Funes), $24.1 \%$ (Rothman) and $30.9 \%$ (Frailty index). Importantly, these studies mainly consisted of non-elective admissions, while our study cohort comprised of elective and non-elective admissions to hospital. However, it may be that this methodology truly underestimates the prevalence of frailty within HES.

Not all frailty syndromes are observed, within HES, to be equally prevalent, nor do they appear to be increasing at the same rate. The observed differences and increase in frailty syndromes in this study (figure 4) may reflect improvements in coding practice within HES due to the introduction of Healthcare Resource Group (HRG V.4 introduced in April 2007) and Payment by Results (since April 2009). The national dementia strategy was also published in 2009. However, this observed rising trend may also reflect a genuine increase in number of diagnosis. Correlation with clinical data sets for comparison is consequently a necessary research priority.

The frailty syndromes are more prevalent in the very elderly $(>85)$, with a rising trend. The exception to this is anxiety and/or depression, where the most prevalent age-band is 75-84 years, which exhibits a declining trend, while the increase in this anxiety and/or depression from 2010 appears to mainly be in the 65-74 age-band, a pattern noted independently by the HSCIC. ${ }^{50}$ Correlation with clinical data sets is warranted to ensure accuracy.

This analysis suggests that coexistence of multiple frailty syndromes is uncommonly coded within HES; even though we used coded frailty syndromes within all 20 of HES diagnostic domains, incomplete coding may still be a cause, as not all morbidities will be acknowledged and coded for each admission, only those deemed relevant to care at that time. However, it has been noted that accumulation of deficit beyond a certain level is incompatible with survival, ${ }^{51}$ and thus multimorbidity would have a ceiling effect. Further investigation on multiple frailty syndromes could be profitable.

Inpatient mortality trends in this population exhibit seasonality with peaks during winter, which persist after adjustment for number of admissions (spells). These peaks, coupled with rising 30-day readmissions (particularly in the very elderly) suggest differences in service provision over the year. A question arises here: is this 

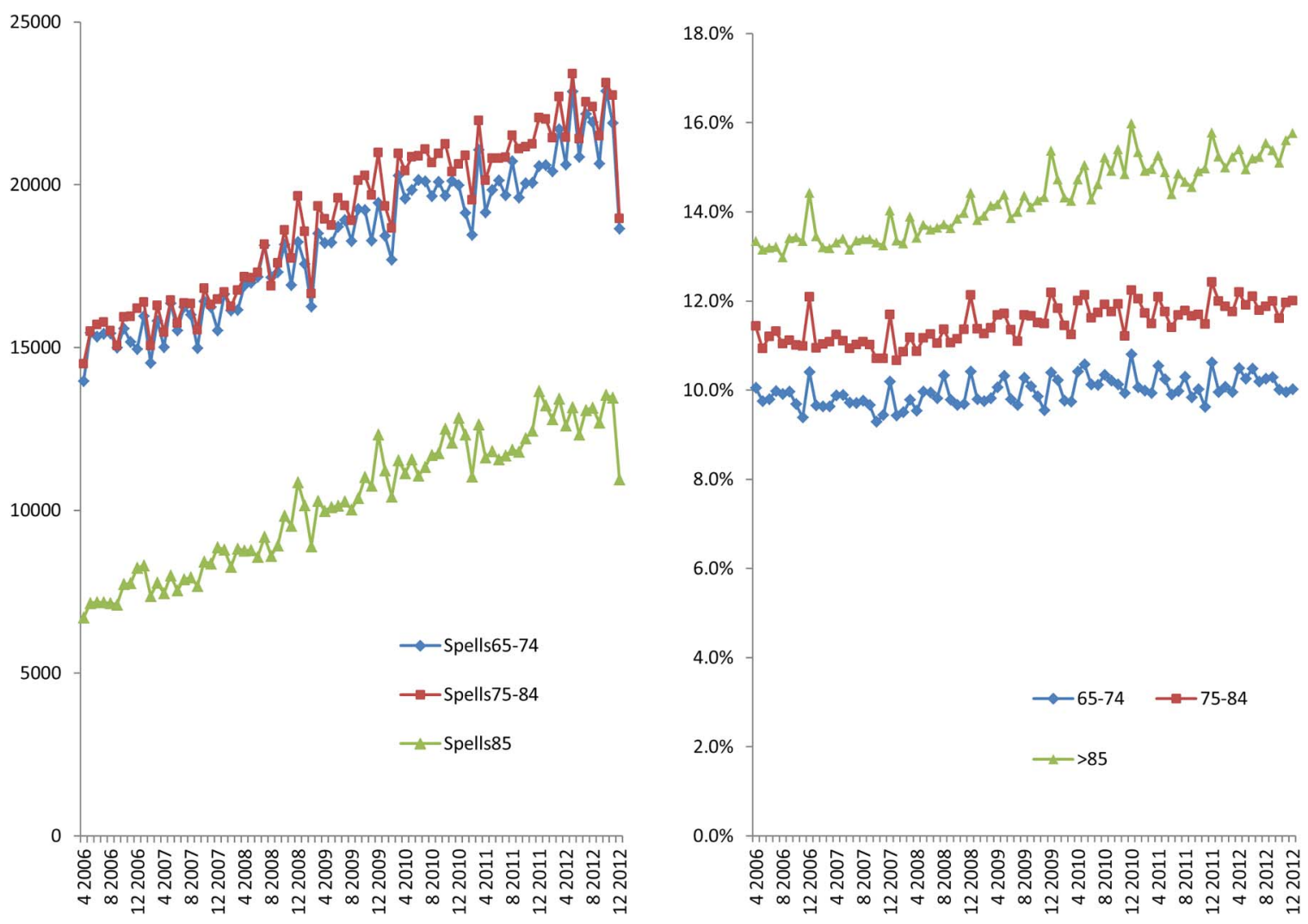

Figure 7 Number and percentage non-elective readmissions in patient $>65$ years admitted to NHS acute providers by age-band. NHS, National Health Service.

seasonality appropriate for the UK population and the provision of care?

Geographic variation in frailty burden appears to be in keeping with known distribution of prevalence of the English elderly population and location of NHS acute provider sites, particularly within urban areas. Healthcare providers and commissioners should consider their local populations when planning services, where frailty may be a larger consideration than other locations. Further study into environmental factors in relation to frailty is a necessary next step.

\section{Limitations}

This study is a retrospective analysis reliant on data coded from hospital data warehouses, and subsequently cleaned into HES. As such, its validity is dependent on accuracy of data coding. Including all 20 diagnostic coding fields may help to mitigate this, but correlation with clinical data sets may be warranted for local investigations. Resultant prevalence rates described may underestimate frailty syndromes in this population.

Anxiety and/or depression was only recently recognised as a geriatric syndrome by the Education Committee Writing Group of the American Geriatrics Society. ${ }^{16}$ It appears to fulfil several criteria that makes it an attractive putative candidate for a frailty syndrome: ${ }^{51}$ poor mental health is often associated with chronic physical deficits, ${ }^{52}$ it appears to increase with age (figure 4), it is associated with adverse outcome, ${ }^{53}$ it is neither to rare or too common (figure 4) Recent study has linked it to frailty ${ }^{52}$ in older persons, though comprehensive study of its relationship to adverse outcomes with relation to frailty is still lacking. Further study, including correlation with clinical data sets, is warranted.

\section{Conclusion}

To our knowledge this study is the first to attempt to use frailty syndromes as an operational definition within an English secondary care data set. While the study is dependent on the accuracy, reliability and retrospective nature of coding within HES, its strengths include being a whole population analysis, with robust trend analysis examining coding reliability. It utilises routinely collected data and is comprehensive in its coding of frailty within all of the diagnostic coding positions in the HES data set. Future studies to correlate with clinical data sets are needed to further investigate the phenomena discovered in this study.

This study provides a methodology to reliably quantify frailty. Applications include the ability to evaluate the effect of interventions over time allowing for health service quality improvement. Geographic analysis allows providers and payers to highlight areas of need, unmet or otherwise for more intelligent targeting of resources, from a public health or clinical perspective. A reliable and quantifiable metric for frailty enables the 
development of risk-prediction models and clinical scoring systems that will aid targeted interventions to vulnerable populations that will benefit most.

Contributors JS conceived study, designed analysis, interpreted results and wrote first draft. AJP designed analysis, interpreted results, contributed to ongoing writing. SS and KD designed analysis. TW designed SPC analysis. DL designed GIS analysis. DB conceived study, designed analysis, interpreted results and contributed to ongoing writing.

Funding This research received no specific grant from any funding agency in the public, commercial or not-for-profit sectors.

Disclaimer This article presents independent research commissioned by the National Institute for Health Research (NIHR) under the Collaborations for Leadership in Applied Health Research and Care (CLAHRC) programme for North West London. The views expressed in this publication are those of the author(s) and not necessarily those of the NHS, the NIHR or the Department of Health. JS received a Research Fellowship award from Chelsea and Westminster Health Charity. SS and KD seconded from Oliver Wyman to support this project. The lead author affirms that the manuscript is an honest, accurate, and transparent account of the study being reported; that no important aspects of the study have been omitted; and that any discrepancies from the study as planned (and, if relevant, registered) have been explained.

Competing interests None declared.

Ethics approval As per Governance Arrangements for Research Ethics Committees (GAfREC), Research limited to secondary use of information previously collected in the course of normal care (without an intention to use it for research at the time of collection), provided that the patients or service users are not identifiable to the research team in carrying out the research.

Provenance and peer review Not commissioned; externally peer reviewed.

Data sharing statement No additional data are available.

Open Access This is an Open Access article distributed in accordance with the terms of the Creative Commons Attribution (CC BY 4.0) license, which permits others to distribute, remix, adapt and build upon this work, for commercial use, provided the original work is properly cited. See: http:// creativecommons.org/licenses/by/4.0/

\section{REFERENCES}

1. United Nations Department of Economics and Social Affairs. Demographic Yearbook 64th issue. 2013;64:1-912.

2. Cornwell J. The Care of Frail Older People with Complex Needs: Time for a revolution. The Sir Roger Bannister Health Summit, LeedsCastle: The King's Fund, 2012.

3. Department of Health. Improving care and saving money: learning the lessons on prevention and early intervention for older people. 2010.

4. Blunt I, Bardsley M, Dixon J. Trends in emergency admissions in England 2004-2009: is greater efficiency breeding inefficiency. London: The Nuffield Trust, 2010.

5. Marengoni A, Angleman S, Melis R, et al. Aging with multimorbidity: a systematic review of the literature. Ageing Res Rev 2011;10:430-9.

6. Barnett K, Mercer SW, Norbury M, et al. Epidemiology of multimorbidity and implications for health care, research, and medical education: a cross-sectional study. Lancet 2012;380:37-43.

7. Rodriguez-Manas L, Feart C, Mann G, et al. Searching for an operational definition of frailty: a Delphi method based consensus statement: the frailty operative definition-consensus conference project. J Gerontol A Biol Sci Med Sci 2013;68:62-7.

8. Fried LP, Tangen CM, Walston J, et al. Frailty in older adults: evidence for a phenotype. J Gerontol A Biol Sci Med Sci 2001;56: M146-56.

9. Ferrucci L, Guralnik JM, Studenski S, et al. Designing randomized, controlled trials aimed at preventing or delaying functional decline and disability in frail, older persons: a consensus report. $J \mathrm{Am}$ Geriatr Soc 2004;52:625-34.

10. Rockwood K, Howlett SE, MacKnight C, et al. Prevalence, attributes, and outcomes of fitness and frailty in community-dwelling older adults: report from the Canadian study of health and aging. J Gerontol A Biol Sci Med Sci 2004;59:1310-17.
11. Ellis G, Whitehead MA, O'Neill D, et al. Comprehensive geriatric assessment for older adults admitted to hospital. Cochrane Database of Systematic Reviews 2011;(7):CD006211.

12. Edmans J, Bradshaw L, Gladman JRF, et al. The Identification of Seniors at Risk (ISAR) score to predict clinical outcomes and health service costs in older people discharged from UK acute medical units. Age Ageing 2013;42:747-53.

13. Wou F, Gladman JR, Bradshaw L, et al. The predictive properties of frailty-rating scales in the acute medical unit. Age Ageing 2013;42:776-81.

14. Isaacs $\mathrm{B}$. The challenge of geriatric medicine. USA: Oxford University Press, 1992

15. Inouye SK, Studenski S, Tinetti ME, et al. Geriatric syndromes: clinical, research, and policy implications of a core geriatric concept. J Am Geriatr Soc 2007:55:780-91.

16. [No authors listed]. Core competencies for the care of older patients: recommendations of the American Geriatrics Society. The Education Committee Writing Group of the American Geriatrics Society. Acad Med 2000;75:252-5.

17. Acute Care Toolkit 3. Acute medical care for frail older people. London: Royal College of Physicians, 2012.

18. Anpalahan M, Gibson SJ. Geriatric syndromes as predictors of adverse outcomes of hospitalization. Intern Med J 2008;38:16-23.

19. Clegg A, Young J, lliffe S, et al. Frailty in elderly people. Lancet 2013;381:752-62.

20. Banerjee J, Conroy S, Cooke MW. Quality care for older people with urgent and emergency care needs in UK emergency departments. Emerg Med J 2013;30:699-700.

21. HES Online: Hospital Episode Statistics. 2013. http://www.hscic.gov. uk/hes (accessed Nov 2014)

22. Health and social care information centre. Methodology to create provider and CIP spells from HES APC data: 1-9, 2014. http://www. hscic.gov.uk/media/11859/Provider-Spells-Methodology/pdf/Spells_ Methodology.pdf (accessed Nov 2014).

23. Bottle A, Aylin P. Comorbidity scores for administrative data benefited from adaptation to local coding and diagnostic practices. $J$ Clin Epidemiol 2011;64:1426-33.

24. Lloyd P, Provost SM. The Health Care Data Guide: Learning from Data for Improvement. John Wiley \& Sons October 2011:1-480.

25. Morley JE, Vellas B, van Kan GA, et al. Frailty consensus: a call to action. J Am Med Dir Assoc 2013;14:392-7.

26. Fried LP, Ferrucci L, Darer J, et al. Untangling the concepts of disability, frailty, and comorbidity: implications for improved targeting and care. J Gerontol A Biol Sci Med Sci 2004;59:M255-63.

27. Theou O, Rockwood MR, Mitnitski A, et al. Disability and co-morbidity in relation to frailty: how much do they overlap? Arch Gerontol Geriatr 2012;55:e1-8.

28. Rockwood K. What would make a definition of frailty successful? Age Ageing 2005;34:432-4.

29. Cesari M, Gambassi G, van Kan GA, et al. The frailty phenotype and the frailty index: different instruments for different purposes. Age Ageing 2014;43:10-12.

30. Blodgett $\mathrm{J}$, Theou O, Kirkland $\mathrm{S}$, et al. Frailty in NHANES: Comparing the frailty index and phenotype. Arch Gerontol Geriatr 2015;60:464-70.

31. Young J, Hood C, Woolley R, et al. Report of the National Audit of Dementia Care in General Hospitals 2011. Royal College of Psychiatrists, 2011.

32. Arora VM, McGory ML, Fung $\mathrm{CH}$. Quality indicators for hospitalization and surgery in vulnerable elders. J Am Geriatr Soc 2007;55(Suppl 2):S347-58.

33. Treml J, Husk J, Lowe D, et al. Falling Standards, Broken Promises. Report of the national audit of falls and bone health in older people 2010. London: Royal College of Physicians, 2011.

34. Wagg A, Harari D, Husk J, et al. National Audit of Continence Care - Combined Organisational and Clinical Report. London: Royal College of Physicians, 2010.

35. Rockwood K, Mitnitski A, Song X, et al. Long-term risks of death and institutionalization of elderly people in relation to deficit accumulation at age 70. J Am Geriatr Soc 2006;54:975-9.

36. Collard RM, Boter $\mathrm{H}$, Schoevers RA, et al. Prevalence of frailty in community-dwelling older persons: a systematic review. J Am Geriatr Soc 2012;60:1487-92.

37. Santos-Eggimann B, Cuenoud P, Spagnoli J, et al. Prevalence of frailty in middle-aged and older community-dwelling Europeans living in 10 countries. J Gerontol A Biol Sci Med Sci 2009;64: 675-81.

38. Syddall H, Roberts HC, Evandrou M, et al. Prevalence and correlates of frailty among community-dwelling older men and women: findings from the Hertfordshire Cohort Study. Age Ageing 2010;39:197-203. 
39. Hubbard RE, Lang IA, Llewellyn DJ, et al. Frailty, body mass index, and abdominal obesity in older people. J Gerontol A Biol Sci Med Sci 2010;65:377-81.

40. Joosten E, Demuynck M, Detroyer E, et al. Prevalence of frailty and its ability to predict in hospital delirium, falls, and 6-month mortality in hospitalized older patients. BMC Geriatr 2014;14:1.

41. Ensrud KE, Ewing SK, Taylor BC, et al. Comparison of 2 frailty indexes for prediction of falls, disability, fractures, and death in older women. Arch Intern Med 2008;168:382-9.

42. Purser JL, Kuchibhatla MN, Fillenbaum GG, et al. Identifying frailty in hospitalized older adults with significant coronary artery disease. J Am Geriatr Soc 2006;54:1674-81.

43. Rockwood K, Stadnyk K, MacKnight C, et al. A brief clinical instrument to classify frailty in elderly people. Lancet 1999;353:205-6.

44. Andela RM, Dijkstra A, Slaets JP, et al. Prevalence of frailty on clinical wards: description and implications. Int $J$ Nurs Pract 2010;16:14-19.

45. Steverink N, Slaets J, Schuurmans $\mathrm{H}$, et al. Measuring frailty: developing and testing of the Groningen Frailty Indicator (GFI). Gerontologist 2001;41:236-7.

46. Ekerstad N, Swahn E, Janzon M, et al. Frailty is independently associated with short-term outcomes for elderly patients with non-ST-segment elevation myocardial infarction. Circulation 2011;124:2397-404.
47. Rockwood K, Song X, MacKnight C, et al. A global clinical measure of fitness and frailty in elderly people. CMAJ 2005;173:489.

48. Evans SJ, Sayers M, Mitnitski A, et al. The risk of adverse outcomes in hospitalized older patients in relation to a frailty index based on a comprehensive geriatric assessment. Age Ageing 2014;43:127-32.

49. McCusker J, Bellavance F, Cardin S, et al. Detection of older people at increased risk of adverse health outcomes after an emergency visit: the ISAR screening tool. J Am Geriatr Soc 1999;47:1229-37.

50. Health and Social Care Information Centre. Anxiety: hospital admissions highest in women in their late 60s. URL: http://www. hscic.gov.uk/article/3916/Anxiety-hospital-admissions-highest-inwomen-in-their-late-60s (accessed Nov 2014)

51. Searle SD, Mitnitski A, Gahbauer EA, et al. A standard procedure for creating a frailty index. BMC Geriatr 2008;8:24.

52. Ni Mhaolain AM, Fan CW, Romero-Ortuno R, et al. Frailty, depression, and anxiety in later life. Int Psychogeriatr 2012;24:1265-74.

53. Dent E, Hoogendijk EO. Psychosocial factors modify the association of frailty with adverse outcomes: a prospective study of hospitalised older people. BMC Geriatr 2014;14:108.

54. Mezuk B, Edwards L, Lohman M, et al. Depression and frailty in later life: a synthetic review. Int $J$ Geriatr Psychiatry 2012;27:879-92. 


\section{Correction}

Soong J, Poots AJ, Scott S, et al. Quantifying the prevalence of frailty in English hospitals. BMJ Open 2015;5:e008456. The corresponding author's email address is incorrect in this paper. The correct address is j.soong@imperial.ac.uk

BMJ Open 2015;5:e008456corr1. doi:10.1136/bmjopen-2015-008456corr1

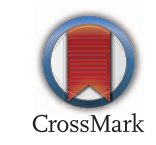

